\title{
Cystic and solitary nodular pulmonary metastases in a patient with low-grade endometrial stromal sarcoma: A case report and literature review
}

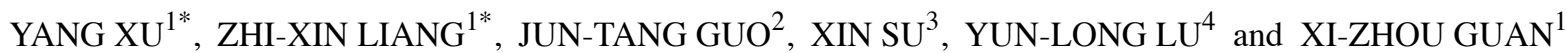 \\ Departments of ${ }^{1}$ Respiratory Diseases and ${ }^{2}$ Thoracic Surgery, Chinese People's Liberation Army General Hospital, \\ Beijing 100853; Departments of ${ }^{3}$ Respiratory Diseases and ${ }^{4}$ Pathology, Hainan Branch of Chinese \\ People's Liberation Army General Hospital, Sanya, Hainan 572013, P.R. China
}

Received April 17, 2018; Accepted April 17, 2019

DOI: $10.3892 / \mathrm{ol} .2019 .10409$

\begin{abstract}
Pulmonary metastases of endometrial stromal sarcoma (ESS) are uncommon and can be difficult to diagnose. The aims of the present study were to investigate the clinical and pathological features, and enhance the awareness of pulmonary metastases in patients with low-grade ESS. The study reports a case of low-grade ESS that resulted in cystic and nodular pulmonary metastases. Furthermore, the PubMed database was searched using 'pulmonary metastases of low-grade endometrial stromal sarcoma' as the key phrase. The literature on pulmonary metastases of low-grade ESS was reviewed and 35 cases were included in the present study. The clinical manifestations, imaging data, pathological features, treatment and prognosis of the 35 previously reported cases and the current case were retrospectively analyzed. The age range of the 36 patients diagnosed with low-grade ESS was 28-65 years. The time period from confirmation of ESS to lung metastases was $1.5-27$ years. In $50 \%$ of the patients, the pulmonary metastases were asymptomatic. The most common pulmonary symptom was dyspnea, followed by chest pain, pneumothorax and coughing. The most common chest imaging presentation was multiple pulmonary nodules, followed by a solitary nodule or mass. Histology was used to
\end{abstract}

Correspondence to: Professor Xi-Zhou Guan, Department of Respiratory Diseases, Chinese People's Liberation Army General Hospital, 28 Fuxing Road, Beijing 100853, P.R. China

E-mail: gxz301@163.com

*Contributed equally

Abbreviations: ESS, endometrial stromal sarcoma; CT, computed tomography; VATS, video-assisted thoracoscopic surgery; CD10, cluster of differentiation 10; ER, estrogen receptor; PR, progesterone receptor; SMA, $\alpha$-smooth muscle actin; CK, creatine kinase

Key words: low-grade endometrial stromal sarcoma, pulmonary metastasis, cystic metastasis, solitary nodular metastases, estrogen receptor, progesterone receptor, hormonal therapy identify that the pulmonary metastases had the pathological features of low-grade ESS. The immunohistochemical results demonstrated strong diffuse immunoreactivity for cluster of differentiation 10, estrogen receptor and progesterone receptor in almost all the specimens. The review of the literature revealed that pulmonary metastases from low-grade ESS are rare but not negligible. Furthermore, the detailed clinical information, imaging findings and immunohistochemical detection are important for making a diagnosis.

\section{Introduction}

Endometrial stromal tumors are rare tumors that originate from the endometrial stroma, accounting for $<2 \%$ of all cases of uterine tumor $(1,2)$. According to clinical, pathological and genetic characteristics, the Fourth Edition of the World Health Organization classification in 2014 (1) divided endometrial stromal tumors into the following four categories: Endometrial stromal nodule, undifferentiated uterine sarcoma, high-grade endometrial stromal sarcoma (ESS) and low-grade ESS.

Low-grade ESS is a rare malignant interstitial tumor, which often occurs in females between 40 and 55 years old (3). Low-grade ESS is an inert tumor with slow growth that has a favorable prognosis (4). In patients with low-grade ESS, $30-50 \%$ of cases present with extrauterine spread at the time of the diagnosis $(3,5-7)$. Distant metastases may develop even if the primary tumor is resected, and the most common location of distant metastases is the lungs, with a reported incidence rate of 7 to $28 \%(8)$.

Pulmonary metastasis of low-grade ESS can manifest as various patterns on computed tomography (CT) images of the chest, which may cause difficulties in making a diagnosis (8-12). Clinical trials and data on the pulmonary metastasis of low-grade ESS are extremely limited. The present study reports one case of pulmonary metastasis of low-grade ESS that was treated at the Chinese PLA General Hospital. In addition, previous literature on the pulmonary metastasis of low-grade ESS was reviewed by searching the PubMed database for pulmonary metastases of low-grade endometrial stromal sarcoma. The clinical manifestations, imaging data, pathological features, treatment and prognosis 
of these cases (the present case and the reported cases retrieved from PubMed) were retrospectively analyzed to investigate the clinical and pathological features, and enhance the awareness of pulmonary metastases in patients with low-grade ESS.

\section{Case report}

A 55-year-old female was admitted to Chinese People's Liberation Army General Hospital (Beijing, China) in March 2017 due to a nodule in the left lung that had been present for 1 year along with recurrent chest tightness, and chest pain that had been occurring for 6 months. In November 2015, the patient had undergone a hysterectomy and bilateral salpingo-oophorectomy to remove low-grade ESS . A pulmonary nodule $(4 \mathrm{~mm})$ was identified in the left upper lobe on the chest $\mathrm{CT}$ scan during the perioperative period, but the patient did not receive any treatment. The patient had experienced chest tightness, shortness of breath and chest pain since August 2016, and the chest CT scan revealed right-sided pneumothorax. The patient was treated with closed thoracic drainage and the pneumothorax subsequently improved. The patient experienced pneumothorax a further three times over the following 6 months. The nodule in the left lung was enlarged and multiple thin-walled cysts in the bilateral lung fields were detected in February 2017.

The patient was admitted to the hospital for further diagnosis and treatment following an existing diagnosis of cystic lung disease and a nodule in the left lung. The physical examination was unremarkable. No moist or dry rales were heard on either side. The heart and abdominal examinations were negative. The superficial lymph nodes were not palpable. The laboratory examination demonstrated a normal white blood cell count. The levels of C-reactive protein and procalcitonin were within normal limits. However, the level of tumor marker cancer antigen 125 was increased compared with the normal levels. The chest CT scan revealed a solid nodule $(2 \times 2.5 \mathrm{~cm})$ in the left upper lung and multiple thin-walled cysts in the bilateral lung fields (Fig. 1).

Video-assisted thoracoscopic surgery (VATS) was performed in March 2017 to make a further diagnosis. Consequently, resections of the solid nodule $(2.5 \times 2 \times 0.8 \mathrm{~cm})$ from the upper lobe (Fig. 2A) and the apex of the left lung, including the thin-walled cysts (Fig. 2B), were performed.

For histopathological and immunohistochemical evaluation of the resected lung nodule, the material from the surgical specimen was fixed in $10 \%$ neutral buffered formalin (cat. no., HT 501128; Sigma-Aldrich; EMD Millipore) for $24 \mathrm{~h}$, at room temperature, embedded in paraffin, cut into $4 \mu \mathrm{m}$-thick sections, and then hematoxylin and eosin (H\&E) and immunohistochemical (IHC) staining were performed.

In H\&E staining, paraffin lung nodule sections were deparaffinized in 2 changes of xylene, $10 \mathrm{~min}$ each, at room temperature. Sections were re-hydrated in 2 changes of alcohol for 5 min each. Alcohol series were as follows: $95 \%$ alcohol for 2 min and $70 \%$ alcohol for $2 \mathrm{~min}$. After washing in running tap water for $5 \mathrm{~min}$, sections were stained with hematoxylin solution (cat, no., 3095771; Sigma-Aldrich; Merck KGaA) for $5 \mathrm{~min}$, at room temperature. After washing in water for $5 \mathrm{~min}$, lung nodule sections were differentiated in $1 \%$ acid alcohol for $30 \mathrm{sec}$ and washed with running tap water for $1 \mathrm{~min}$.
Slides were stained with 5\% eosin solution (cat, no. 318906; Sigma-Aldrich; Merck KGaA) for 1-3 min, at room temperature. After washing in running tap water for $5 \mathrm{~min}$, lung nodule was dehydrated through 95\% alcohol, 2 changes of absolute alcohol for $5 \mathrm{~min}$ each. Finally, lung nodule sections were cleared in 2 changes of xylene for $5 \mathrm{~min}$ each, and mounted with xylene based mounting medium. H\&E staining reaction was observed using a standard light microscope (Leica, DM2000; magnification 10x10; Leica Microsystems GmbH).

In IHC staining, the EnVision two-step immunohistochemical staining technique (13) was used and the visualization system was 3,3'-diaminobenzidine tetrahydrochloride (DAB). Sections were dewaxed and hydrated. After endogenous peroxidase blocking with EnVision $\mathrm{Flex}^{+}$(cat, no., K8002; Dako; Agilent Technologies, Inc.) for $5 \mathrm{~min}$, at room temperature, the sections were incubated with primary antibodies at an optimal dilution (Table I), for $30 \mathrm{~min}$, at room temperature. After washing with PBS for $10 \mathrm{~min}$, the sections were incubated with a secondary antibody EnVision ${ }^{\mathrm{TM}}$ (cat, no., K8009; Dako; Agilent Technologies, Inc.) for $30 \mathrm{~min}$ at room temperature. After being washed with water, signal visualization was performed with 3-3'diaminobenzidine DAB medium (D7034; Sigma-Aldrich; Merck KGaA), for $5 \mathrm{~min}$ at room temperature. Finally, the sections were washed and mounted. The immunohistochemical reaction was interpreted using a standard light microscope (Leica, DM2000; magnification 10x10; Leica Microsystems GMBH).

Upon pathological examination, the resected lung nodule was determined to be a short spindle cell malignant tumor. Hematoxylin and eosin staining indicated a proliferation of oval or spindle cells and scattered arterioles (Fig. 3). Immunohistochemical examinations of the tumor tissues revealed that they were strongly positive for cluster of differentiation 10 (CD10), estrogen receptor (ER) and progesterone receptor (PR). Furthermore, the tumor cells demonstrated positivity for vimentin and $\mathrm{Bcl}-2$, and were negative for $\alpha$-smooth muscle actin, desmin, creatine kinase (CK), S-100 and inhibin (Fig. 3). The immunophenotype supported metastatic stromal sarcoma of the uterus. A cystic specimen was observed in the left upper lobe of the lung and the wall of the cyst was covered with oval cell tumors. The cystic specimen was positively immunoreactive for ER, PR and CD10 (Fig. 4). The results of the histological and immunohistochemical examinations of both the resected lung nodule and the thin-walled cyst specimens demonstrated that the low-grade ESS (the original uterine tumor) had metastasized to the lungs.

After establishing the diagnosis, treatment with megestrol was initiated, which was administered orally at a dose of $320 \mathrm{mg} /$ day. Following 2 months of treatment, a number of the small cysts disappeared, although the majority of them remained unchanged. To date, the patient has continued with the megestrol regimen and no new pulmonary metastatic lesions have been identified.

\section{Literature review}

Materials and methods. The PubMed database was searched using 'pulmonary metastases of low-grade endometrial stromal sarcoma' as the key phrase and the full text results were downloaded. The inclusion criteria were as follows: i) Female 

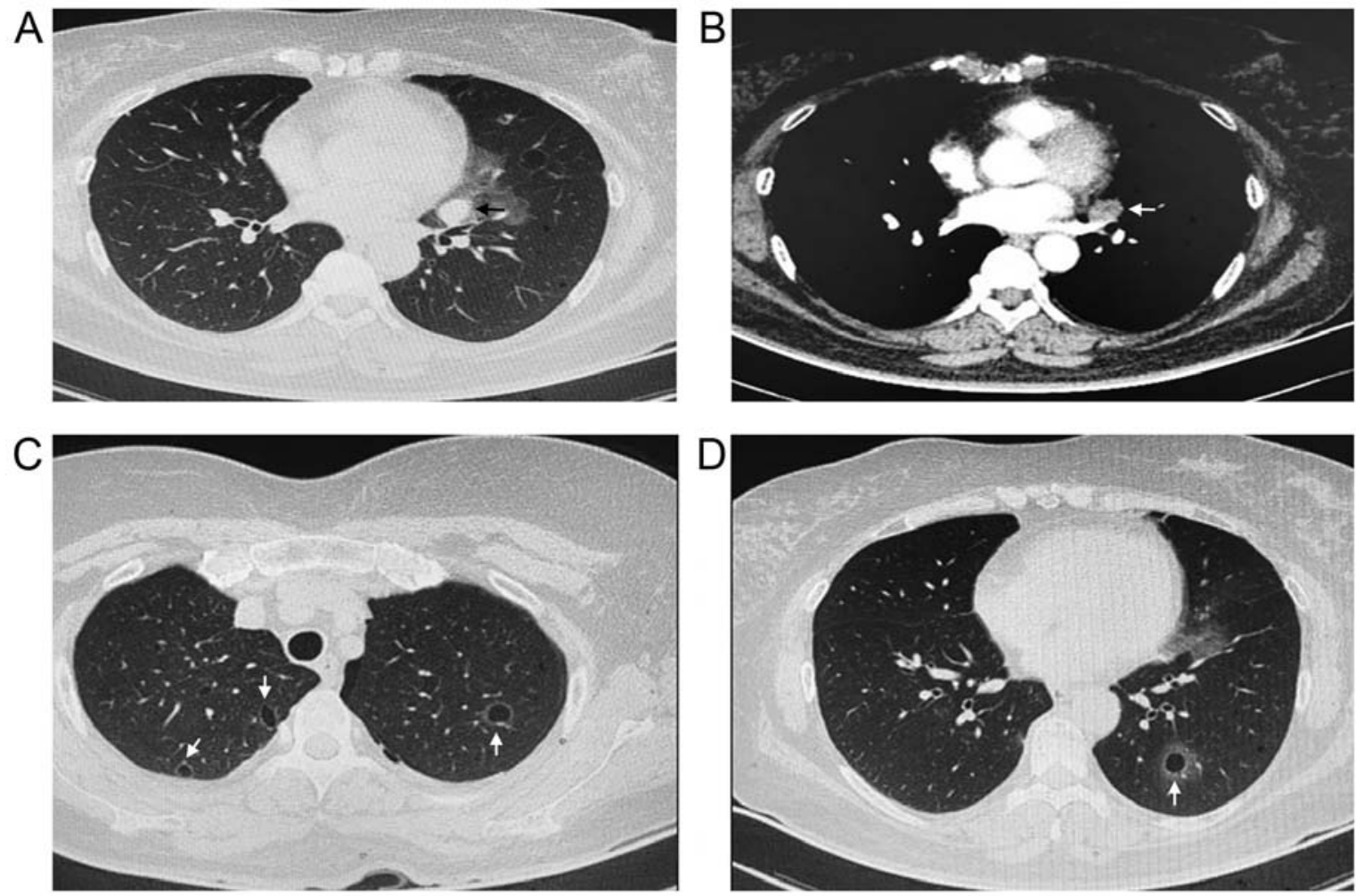

Figure 1. Chest CT scans for the patient with pulmonary metastasis of low-grade endometrial stromal sarcoma. (A) Lung window and (B) mediastinal window CT scans revealed a solid nodule $(2 \times 2.5 \mathrm{~cm})$ in the left upper lung, indicated by the arrow. (C and D) Chest CT scans revealed multiple thin-walled cysts in the bilateral lung fields, indicated by the white arrows. CT, computed tomography.
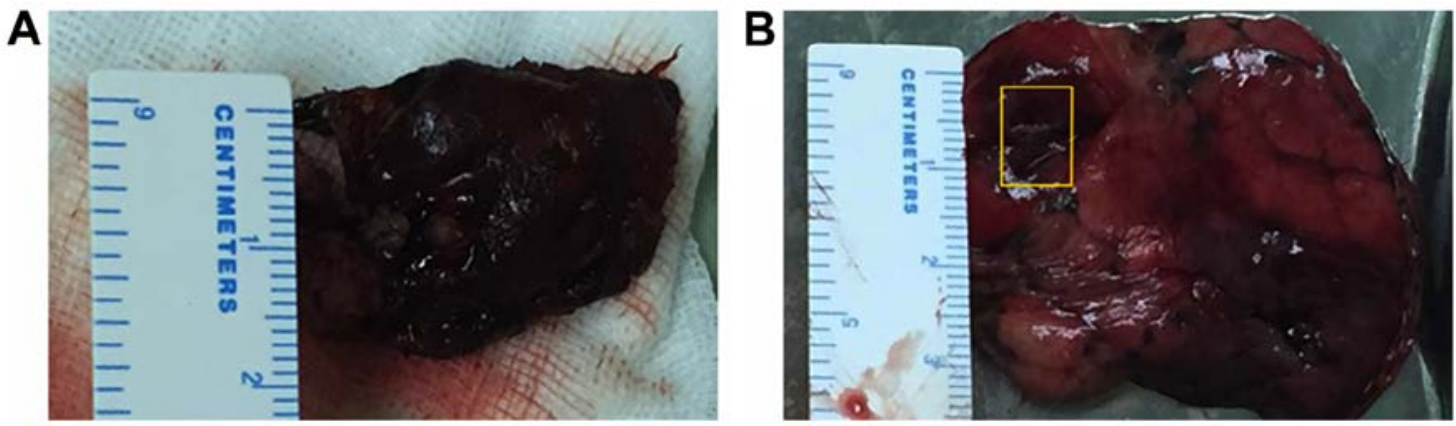

Figure 2. VATS can be performed to make a further diagnosis. Resections of the solid nodule from the upper lobe and the apex of the left lung, including the thin-walled cysts, were performed. (A) Resection of the solid nodule $(2.5 \times 2 \times 0.8 \mathrm{~cm})$ in the upper lobe of the left lung by VATS. (B) Resection of the apex of the left lung, including the thin-walled cysts, by VATS. The yellow rectangle indicates a cyst. VATS, video-assisted thoracoscopic surgery.

patients (without age restriction) with a history of pathologically confirmed low-grade ESS; ii) pathologically confirmed pulmonary metastasis of low-grade ESS that had occurred at the time of the initial diagnosis of low-grade ESS or during follow-up; ii) the article contained clear and specific descriptions of the symptoms, imaging data, pathological features, treatment and prognosis; and ii) the article was published in English. No date limit was applied. Duplicate reports and those that did not meet the inclusion criteria were excluded.

A total of 35 cases described in articles that were retrieved from the PubMed database were included in the present study $(8-12,14-26)$. The present study retrospectively analyzed the clinical manifestations, imaging data, pathological features, treatment and prognosis of the 36 cases of pulmonary metastases of low-grade ESS, which include the 35 reported cases and the present case. The 36 cases are summarized in Table II.

Results. The 36 patients diagnosed with low-grade ESS had an age range of 28-65 years. Among them, 25 patients $(69.4 \%)$ were initially diagnosed with low-grade ESS, and 11 patients $(30.6 \%)$ were initially misdiagnosed as not having low-grade ESS and then received a modified diagnosis of low-grade ESS during treatment. A total of 9 patients $(25 \%)$ had undergone a hysterectomy and bilateral adnexal resection, 25 patients $(69.4 \%)$ had undergone a hysterectomy alone, 1 patient $(2.8 \%)$ had undergone tumor resection and 1 patient (2.8\%) had no history of surgery as the patient refused surgery. Pulmonary metastases were identified in 5 patients $(13.9 \%)$ at the time of diagnosed with low-grade ESS, and in 31 patients $(86.1 \%)$ 
Table I. Primary antibodies used in immunohistochemical analysis.

\begin{tabular}{lcll}
\hline Antibody & Dilution & Catalogue number & \multicolumn{1}{c}{ Supplier } \\
\hline CD10 & $1: 00$ & ab227659 & Abcam, Cambridge, MA, USA \\
ER & $1: 50$ & sc787 & Santa Cruz Biotechnology, CA, USA \\
PR & $1: 50$ & sc810 & Santa Cruz Biotechnology, CA, USA \\
Vimentin & $1: 200$ & ab92547 & Abcam, Cambridge, MA, USA \\
Bcl-2 & $1: 50$ & sc509 & Santa Cruz Biotechnology, CA, USA \\
Inhibin & $1: 50$ & ab14087 & Abcam, Cambridge, MA, USA \\
Desmin & $1: 50$ & sc23879 & Santa Cruz Biotechnology, CA, USA \\
$\alpha$-SMA & $1: 200$ & A5228 & Sigma-Aldrich, Saint Louis, MO, USA \\
CK & $1: 100$ & Ab108388 & Abcam, Cambridge, MA, USA \\
S-100 & $1: 800$ & ab52642 & Abcam, Cambridge, MA, USA \\
\hline
\end{tabular}

CD10, cluster of differentiation 10; ER, estrogen receptor; PR, progesterone receptor; CK, creatine kinase.
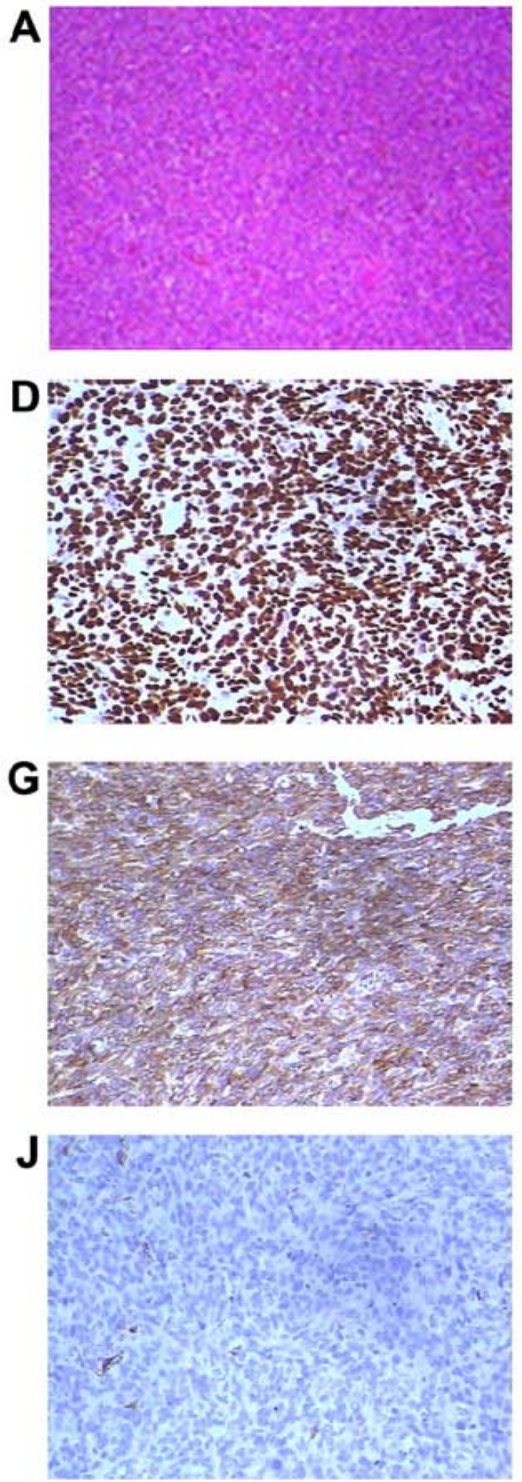
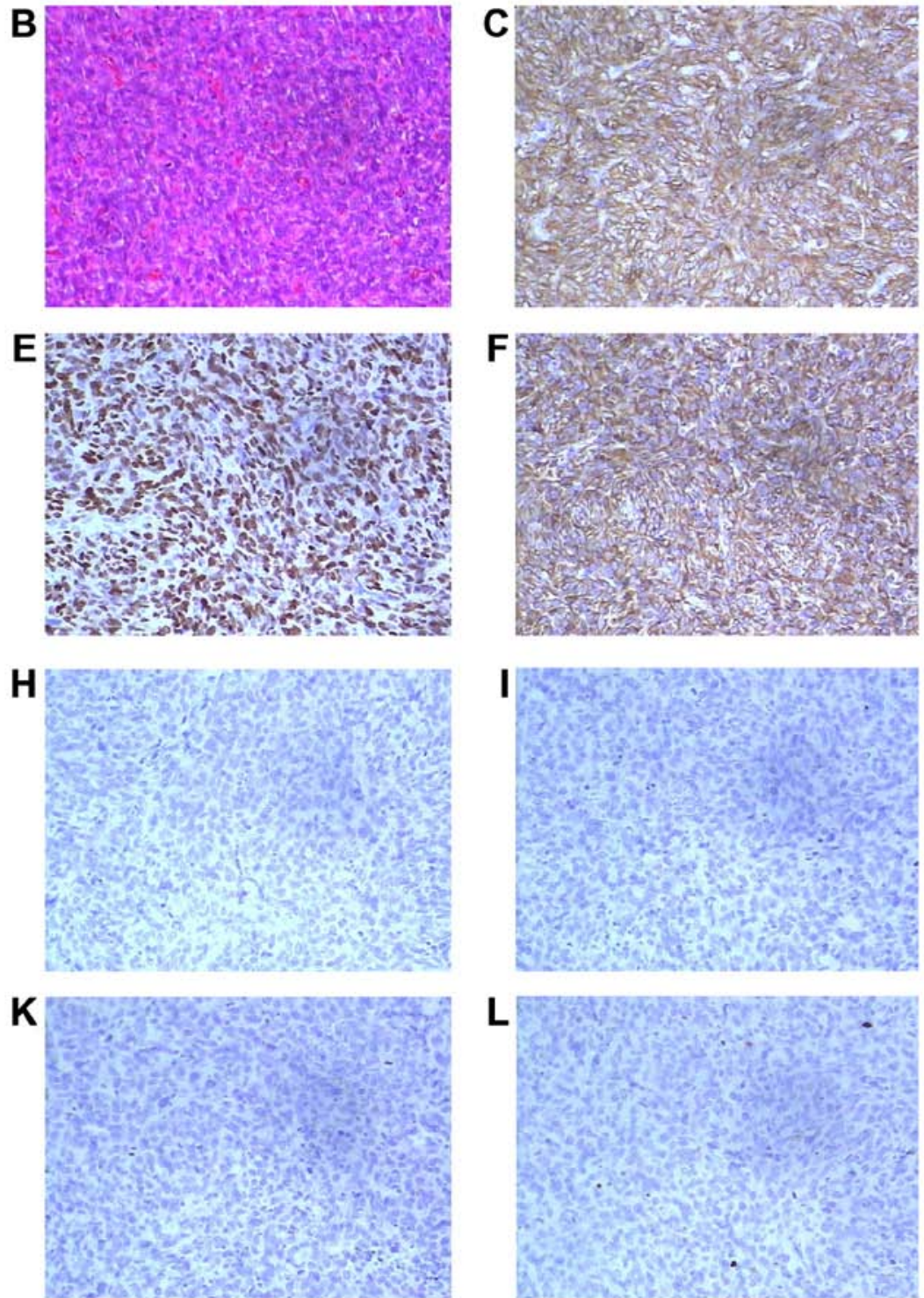

Figure 3. Pathological staining of the resected lung nodule. Upon pathological examination, the resected lung nodule in the left lung was determined to be a short spindle cell malignant tumor. The immunophenotype supported metastatic stromal sarcoma of the uterus. Hematoxylin and eosin staining at magnifications of (A) x100 and (B) revealed an oval or spindle cell proliferation and scattered arterioles. The tumor cells demonstrated positivity for (C) cluster of differentiation 10, (D) estrogen receptor, (E) progesterone receptor, (F) vimentin and (G) Bcl-2. The tumor cells were negative for (H) inhibin, (I) desmin, (J) $\alpha$-smooth muscle actin, (K) creatine kinase and (L) S-100. Magnification, x200. 
A

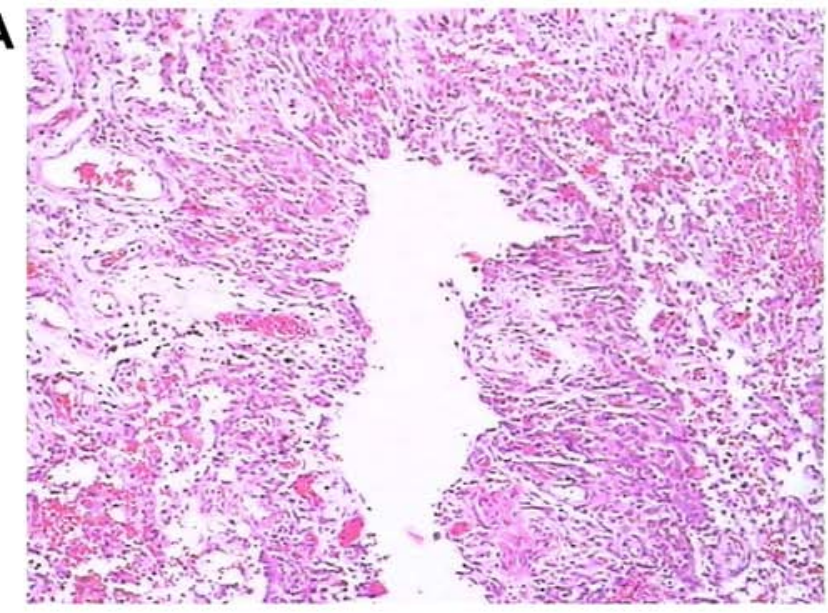

C

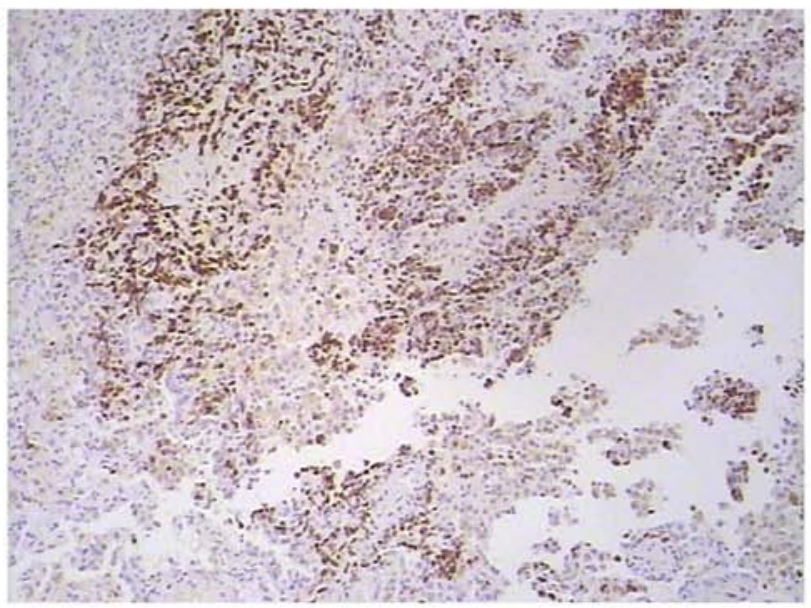

B
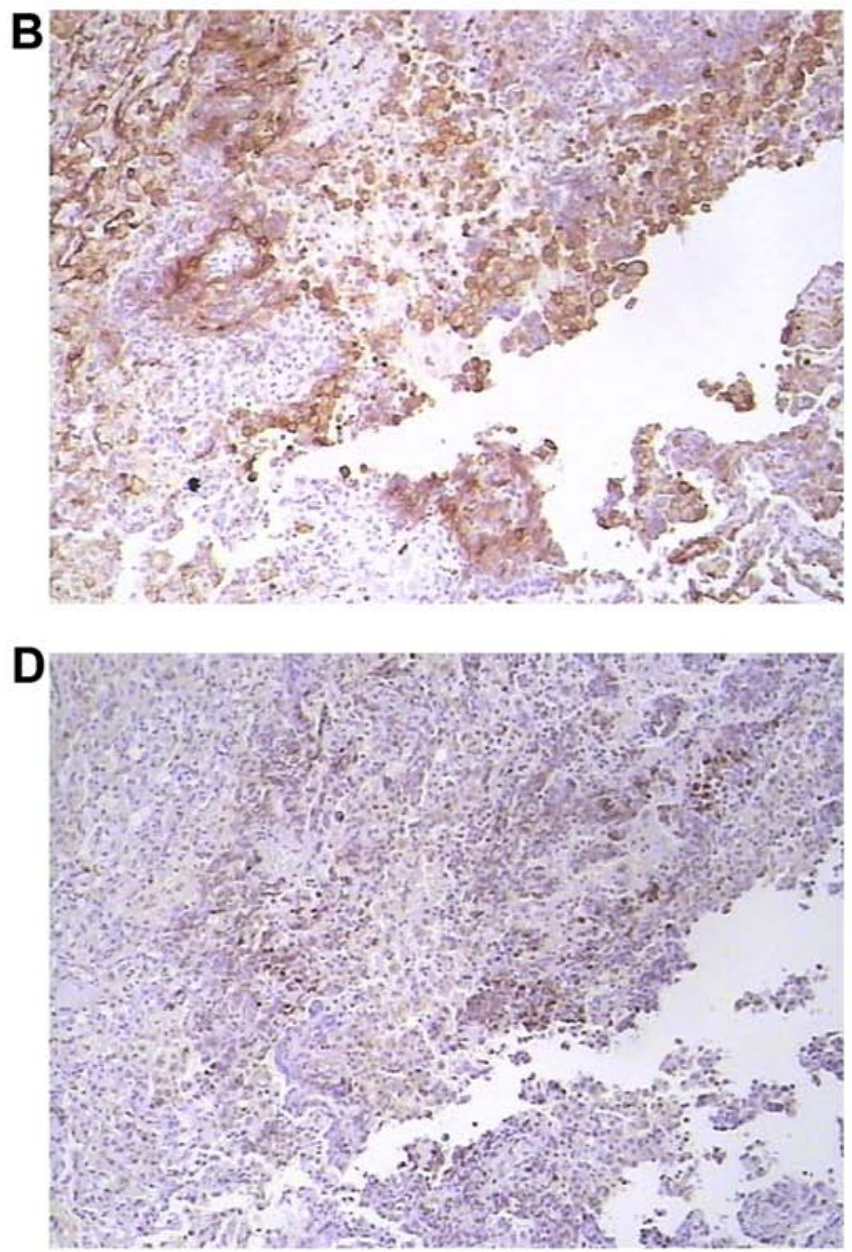

Figure 4. Pathological staining of the cystic specimen. Upon pathological examination of the cystic specimen from the apex of left lung, it was observed that the wall of the cyst was covered with oval cell tumors. The immunophenotype supported metastatic stromal sarcoma of the uterus. (A) Hematoxylin and eosin staining results indicated that the wall of the cyst was covered with oval cell tumors (magnification, x100). The tumor cells demonstrated positivity for (B) cluster of differentiation 10, (C) estrogen receptor and (D) progesterone receptor. Magnification, x100.

following gynecological surgery. The time period from confirmation of low-grade ESS to lung metastases was 1.5-27 years.

Of the 36 patients with pulmonary metastases of low-grade ESS, dyspnea was experienced by 9 patients $(25.0 \%)$, chest pain was reported by 8 patients $(22.2 \%)$, pneumothorax was identified in 7 patients (19.4\%), coughing was experienced by 6 patients $(16.7 \%)$, hemoptysis was presented by 2 patients $(5.6 \%)$ and 18 patients $(50 \%)$ were asymptomatic. The most common pulmonary symptom reported was dyspnea, followed by chest pain, pneumothorax and coughing.

Among the 36 patients with pulmonary metastases of low-grade ESS, 18 patients $(50 \%)$ presented with multiple pulmonary nodules, 6 patients $(16.7 \%)$ had a solitary nodule or mass, 6 patients $(16.7 \%)$ exhibited cystic lesions and 2 patients $(5.6 \%)$ had a reticular formation. Furthermore, 2 patients $(5.6 \%)$ had multiple nodules with pleural effusion, 2 patients $(5.6 \%)$ exhibited multiple pulmonary nodules with cystic lesions, 1 patient $(2.8 \%)$ presented with a solitary nodule with cystic lesions and 1 patient $(2.8 \%)$ had multiple nodules with cystic lesions and cavities.

The histology results for the 36 patients with pulmonary metastases of low-grade ESS demonstrated that the lung lesions were composed of short spindle cells arranged in ill-defined whorls that were centered on numerous uniform arterioles. The neoplastic cells were small with unremarkable nuclear features and sparse cytoplasm. One tumor contained areas of fibroblastic differentiation. Epithelioid features characterized by sex cord-like differentiation were observed in 3 patients. The immunohistochemical results revealed strong diffuse immunoreactivity for ER and PR in almost all of the cases of pulmonary metastasis. There was positive immunoreactivity for CD10 and vimentin and negative immunoreactivity for CD45, CK and S-100 in the majority of the specimens.

Pulmonary surgery in the form of a wedge resection and/or lobectomy was performed in 21 patients. Among them, 5 patients received hormonal therapy following pulmonary surgery and 3 patients received chemotherapy following pulmonary surgery. A total of 8 patients received hormonal therapy only and 2 patients received chemotherapy only. Only 1 patient received chemotherapy following hormonal therapy. In total, 4 patients did not receive any treatment.

Follow-up data were available for 33 patients. Among them, 6 patients succumbed to the disease. A total of 14 were alive with no evidence of disease and 13 were alive with evidence of stable disease. 


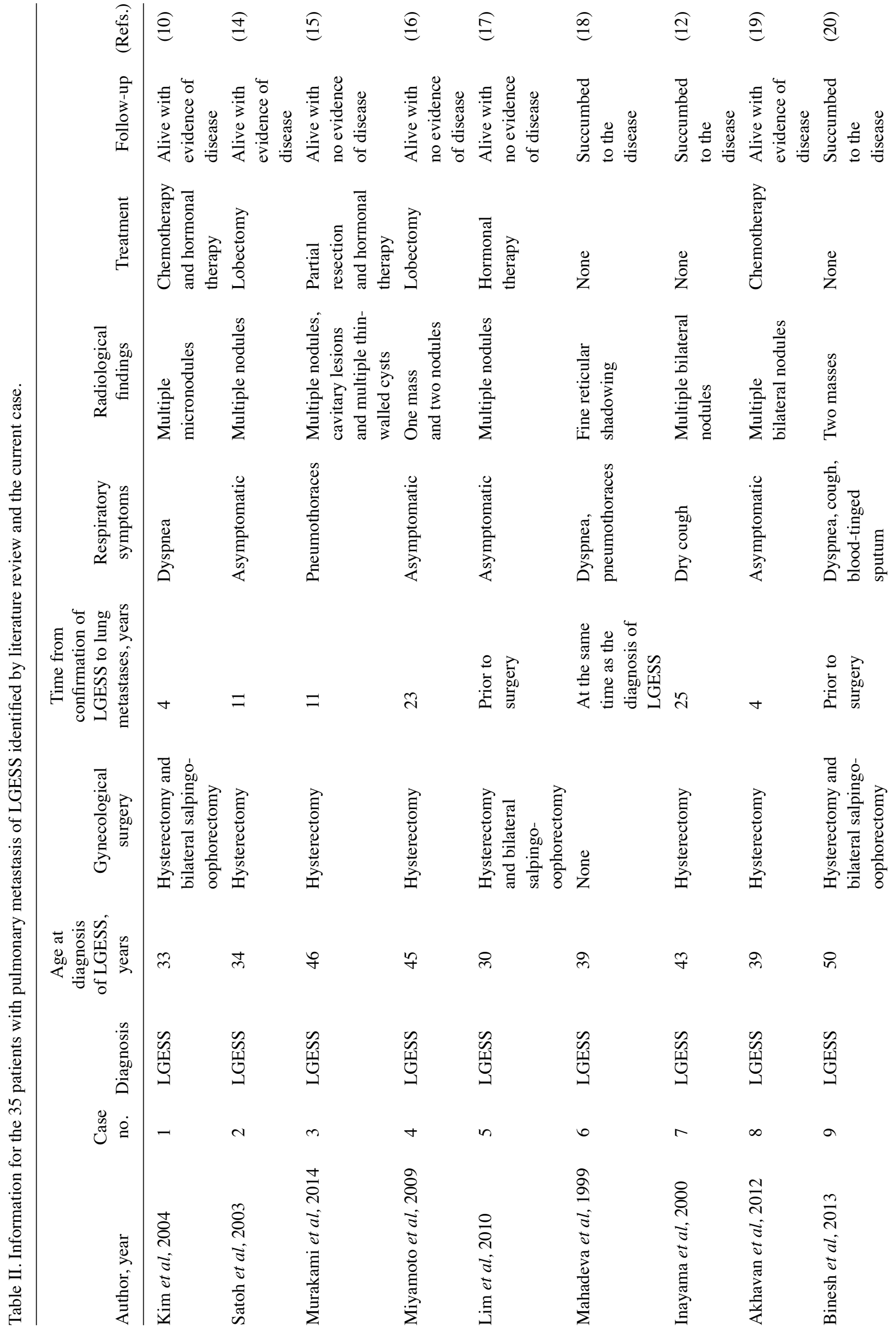




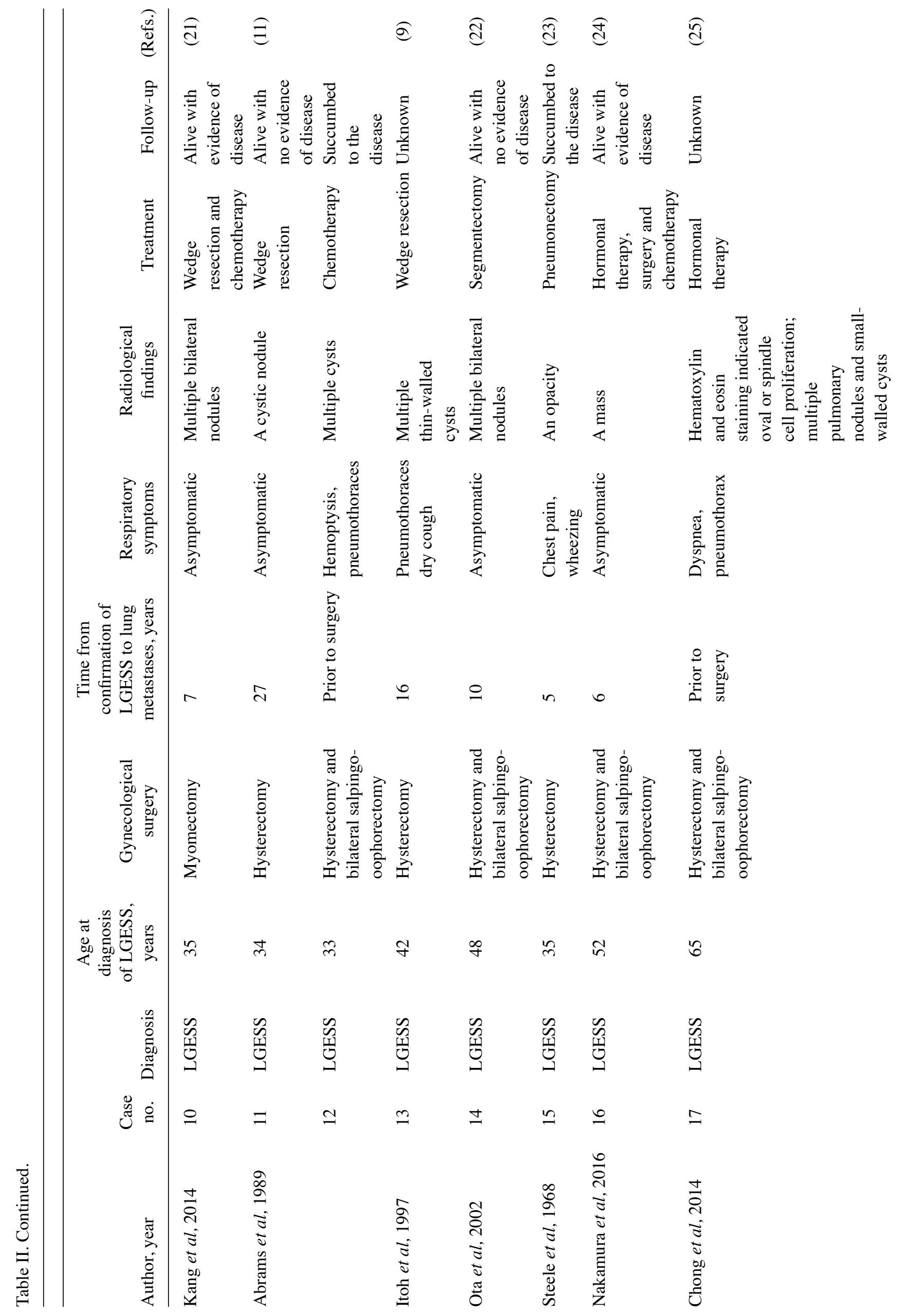


产|

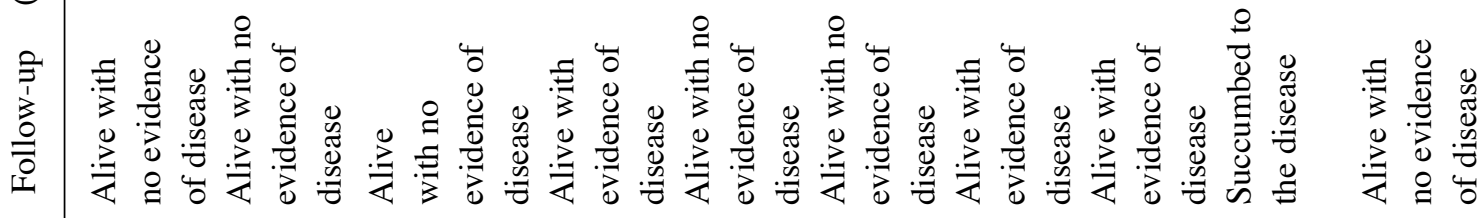

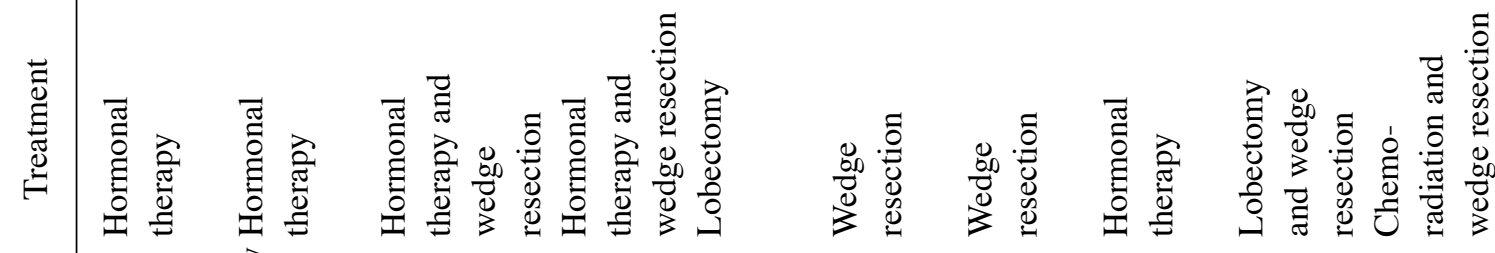

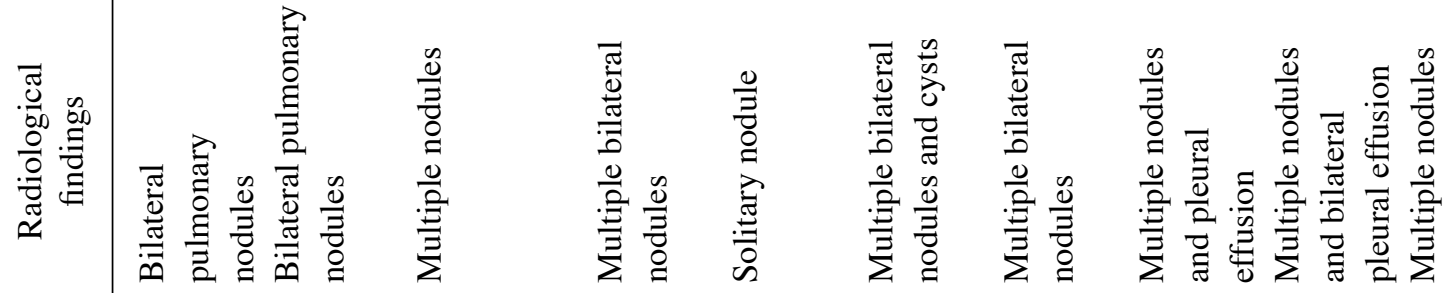

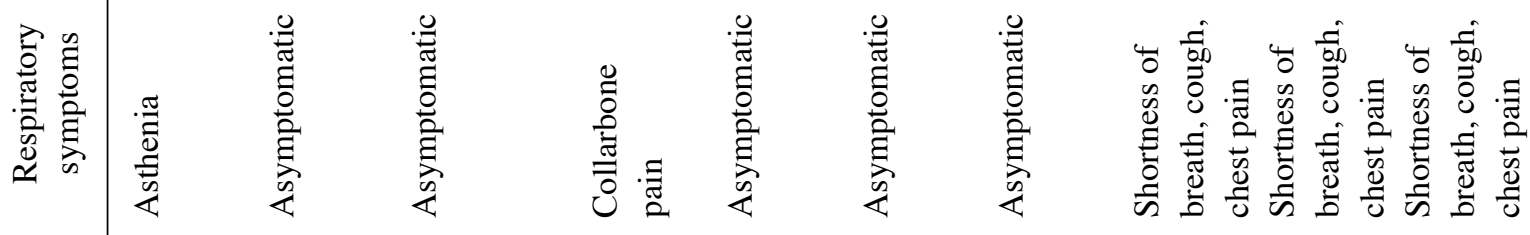

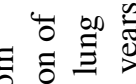

음

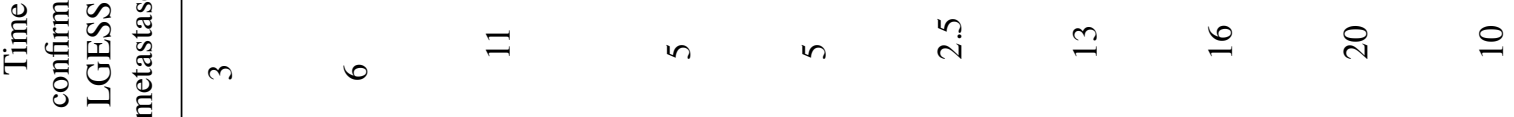

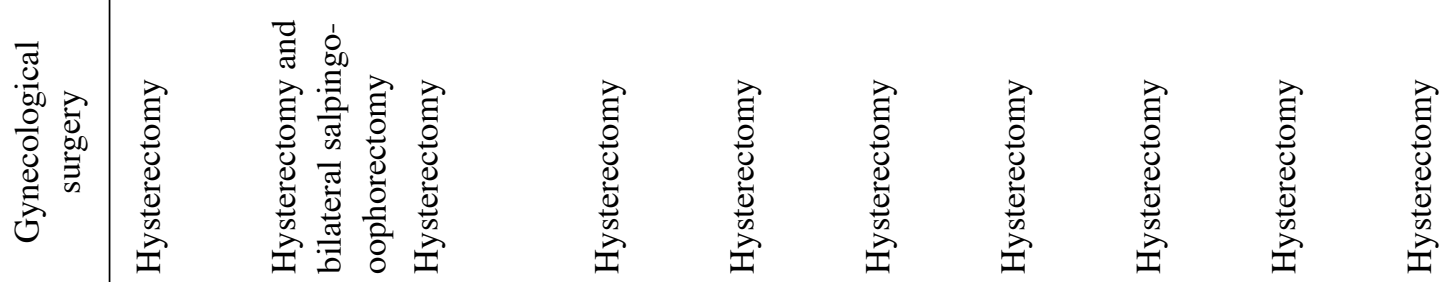

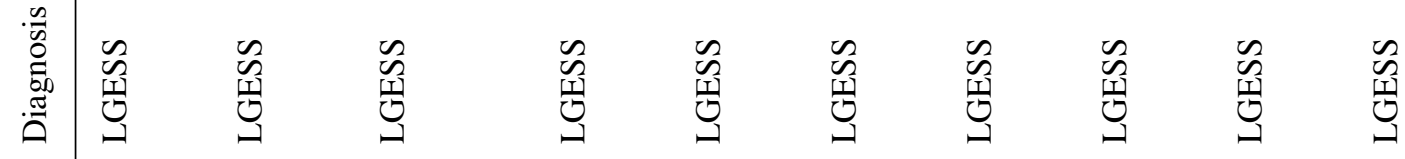

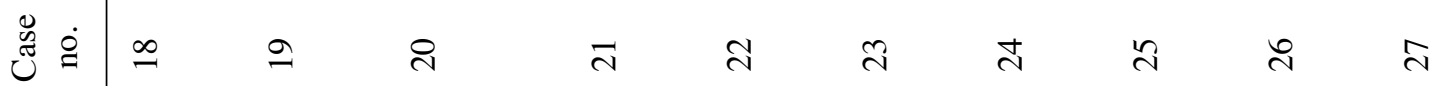




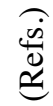

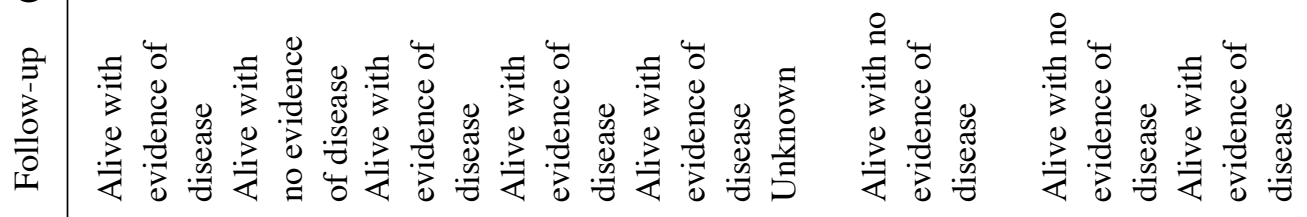

离

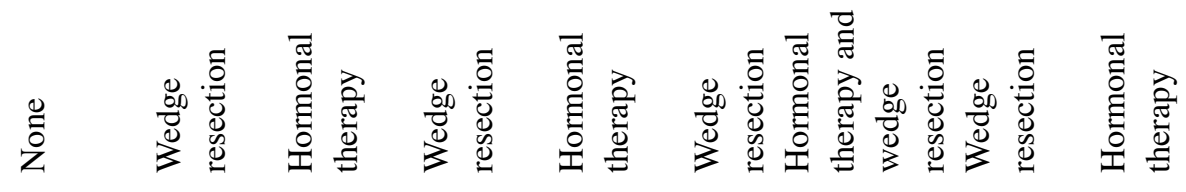

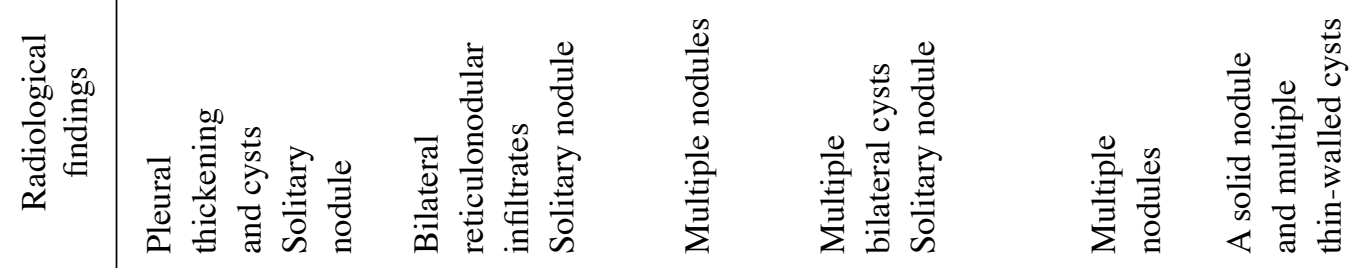

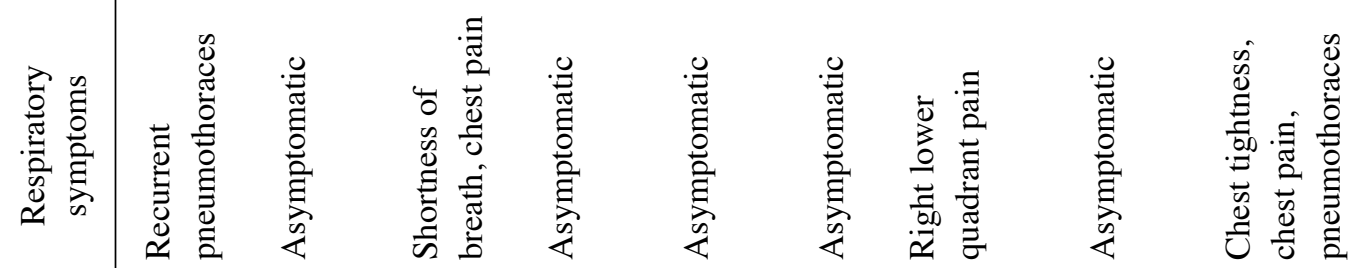

घี

웅

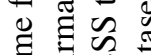

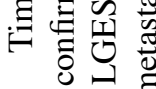

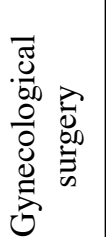

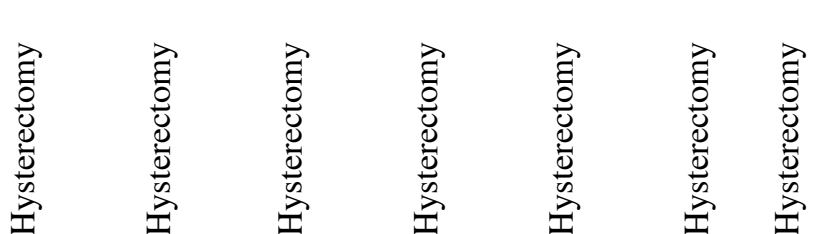

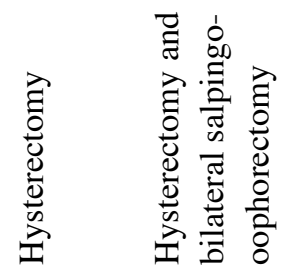

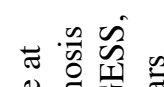

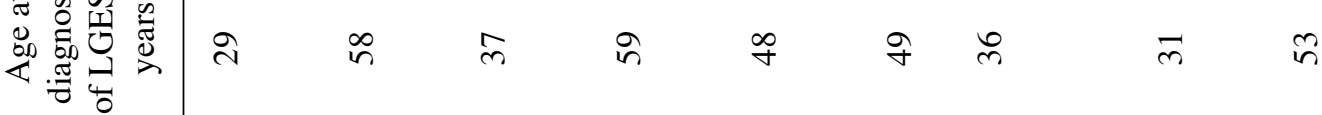

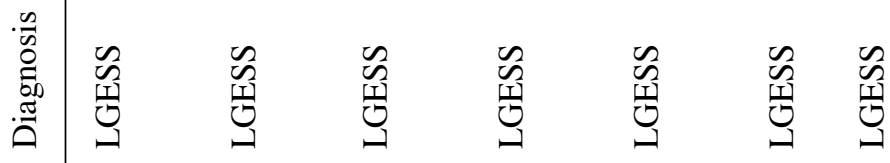

告

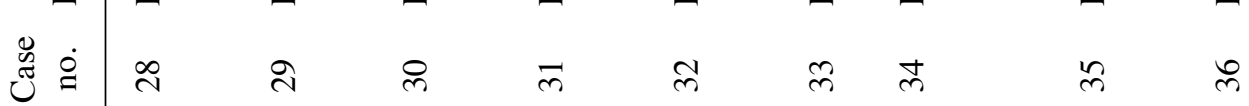




\section{Discussion}

The present study describes the case of a female with metastatic low-grade ESS who presented with simultaneous cystic and solitary nodular lesions via chest CT imaging. The patient had undergone a hysterectomy for low-grade ESS of the uterus 1.5 years previously. The simultaneous presentation of cystic and nodular lesions made the diagnosis problematic. VATS was conducted for further diagnosis and, consequently, resections of the solid nodule and the apex of the left lung, including the thin-walled cysts, were performed. The pathological examination of the resected lung specimens revealed a proliferation of tumor cells with oval-shaped nuclei in both the nodular portion and the cystic lesions. The cystic and solitary nodular lesions were considered to be pulmonary metastasis of low-grade ESS.

To investigate the clinical and pathological features, and enhance the awareness of pulmonary metastases in patients with low-grade ESS, the 35 previously reported cases identified in the literature and the current case were further reviewed. Among these cases, the interval from hysterectomy to the identification of pulmonary metastasis ranged from 1.5 to 27 years. Patients with pulmonary metastases of low-grade ESS usually have no specific symptoms. A number of patients have pulmonary metastasis at the initial diagnosis of low-grade ESS and this may be accompanied by corresponding respiratory symptoms $(3,6,7)$. In the present study, the most common pulmonary symptom was dyspnea, followed by chest pain, pneumothorax and coughing. Asymptomatic pulmonary metastases were identified in 50\% of the patients.

Pulmonary metastasis of low-grade ESS can manifest as various patterns on CT scans (8). The present study identified that the most common pattern of pulmonary metastatic low-grade ESS was multiple pulmonary nodules, which was observed in $50 \%$ of the patients reviewed. Unusual presentations identified in the present study included a solitary nodule, bilateral reticulonodular infiltrates and spontaneous pneumothoraces associated with predominantly cystic lesions. Although the majority of patients possessed multiple nodules, $16.7 \%$ presented with a solitary nodule. Therefore, if a patient has a history of low-grade ESS, the presence of a solitary nodule on a chest CT scan should be closely monitored for lung metastasis. Cystic metastases were identified in $16.7 \%$ of the patients with pulmonary metastasis of ESS that were reviewed in the present study. The thin-walled cysts may have been caused by the proliferation of ESS cells in the airways. Tumor invasion in the bronchial valve leads to pulmonary cysts. A solitary nodule and cysts are individually known to reflect pulmonary metastasis of low-grade ESS; however, the coexistence of these imaging features is rare and can contribute to difficulty in making a diagnosis (8). The present study reports a case of metastatic low-grade ESS that simultaneously presented cystic and solitary nodular lesions via a chest CT scan. The mechanism underlying the coexistence of multiple lesions requires investigation.

At present, the diagnosis of pulmonary metastasis of low-grade ESS is based on the histopathological results (1). Histology is used to confirm that the pulmonary metastasis has the pathological features of low-grade ESS.
Microscopically, low-grade ESS tumor cells are small and consistently shaped, with short fusiform nuclei, swirled around spiral arteriole-like vessels (1). Tumor cells are similar to endometrial stromal cells in the proliferative phase, with minimal cell atypia and usually low mitotic activity ( $<5$ mitoses $/ 10$ high power fields). The typical microscopic feature of the tumor is tongue-like invasion of the muscular layer and lymphatic vessels (8).

Immunohistochemical analysis of low-grade ESS reveals positive expression of CD10, ER and PR. CD10 exhibits high sensitivity and poor specificity, and requires staining with more than two smooth muscle markers, including desmin, SMA or h-caldesmon, to make a definite diagnosis (1). As low-grade ESS can be accompanied by smooth muscle differentiation, smooth muscle markers may be focal positive (8). Hwang et al (27) revealed that the combined application of CD10, ER, PR, h-caldesmon and transgelin successfully distinguishes low-grade ESS from uterine leiomyosarcoma; the identification of positive expression of CD10, ER and PR, and negative expression of $\mathrm{h}$-caldesmon and transgelin increases the diagnostic accuracy for low-grade ESS.

Previous studies have expanded the understanding of the molecular features of ESS. In low-grade ESS, the most common chromosomal translocation is $\mathrm{t}(7 ; 17)(\mathrm{p} 15 ; \mathrm{q} 21)$, which results in the fusion of JAZF1 and SUZ12 genes (28). Other gene fusions include JAZF1-PHF1, EPC1-PHF1, MEAF6-PHF1, ZC3H7-BCOR and MBTD1-CXorF67 (29-32). These cytogenetic changes may be associated with pathogenesis, and can be detected by fluorescence in situ hybridization and reverse transcription-polymerase chain reaction. These aforementioned molecular features may contribute to the diagnosis of morphologically unclear cases.

At present, the treatment for patients with low-grade ESS predominantly consists of surgery (33). The basic surgery for stage I-II low-grade ESS involves a total hysterectomy and bilateral adnexectomy, while patients with advanced stage (III-IV) low-grade ESS can be treated with tumor cell inactivation therapies. Postoperative adjuvant therapies for low-grade ESS can be of great value. The 2016 National Comprehensive Cancer Network Clinical Practice Guidelines for Uterine Tumors (34) recommend that only observation or hormone therapy should be conducted in patients with stage I low-grade ESS, hormone therapy with or without tumor-targeted radiotherapy should be performed in patients with stages II-IVA, and hormone therapy with or without palliative radiotherapy should be conducted in those with stage IVB.

Low-grade ESS has a high reoccurrence rate, and the optimal treatment for low-grade ESS with pulmonary metastasis has not yet been established. It has been reported that patients could benefit from further surgery, including the resection of distant metastatic lesions (35). In the cases reviewed in the present study, the surgeries were performed in the form of wedge resection and/or lobectomy, and the majority of patients exhibited a good prognosis. As low-grade ESS is sensitive to hormone therapy, hormone therapy is also recommended for patients with low-grade ESS that has recurred $(34,36)$. The recommended hormone therapy drugs include megestrol, medroxyprogesterone and aromatase inhibitors, and gonadotropin-releasing hormone analogs can 
also be used (34). Due to the lack of prospective studies, the optimal dosage, drugs and treatment time of hormone therapy are not clear. Low-grade ESS demonstrates a low response rate to chemotherapy, so chemotherapy is only considered when hormone therapy is ineffective (33). In addition, previous studies have reported a number of potential therapeutic targets for low-grade ESS, including platelet-derived growth factor receptor, vascular endothelial growth factor receptor and histone deacetylases (37); however, their clinical value requires further investigation and confirmation.

In conclusion, the present study reports a case of pulmonary metastatic low-grade ESS that simultaneously presented as cystic and solitary nodular lesions. The coexistence of these imaging features therefore indicates pulmonary metastasis of low-grade ESS. The literature review demonstrated that pulmonary metastases of low-grade ESS are not common but should be disregarded. The clinical manifestations are not specific and diagnosis is often difficult. The combination of clinical history, imaging results and histological findings is essential for the diagnosis of low-grade ESS with pulmonary metastasis. A combination of surgery and adjuvant therapy may improve the treatment outcome. As a rare disease, there is a lack of large sample research data on low-grade ESS, and the optimal treatment strategy requires further investigation.

\section{Acknowledgements}

Not applicable.

\section{Funding}

No funding was received.

\section{Availability of data and materials}

All data generated or analyzed during the present study are included in this published article.

\section{Authors' contributions}

YX and ZL made substantial contributions to the analysis and interpretation of patient data, and YX was a major contributor in writing the manuscript. XG made substantial contributions to the conception and design of the study. Video-assisted thoracoscopic surgery was performed by JG. YL performed the pathological examination. XS made contributions to the acquisition of imaging data. All authors read and approved the final manuscript.

\section{Ethics approval and consent to participate}

Not applicable.

\section{Patient consent for publication}

The patient provided written informed consent for publication.

\section{Competing interests}

The authors declare that they have no competing interests.

\section{References}

1. Ali RH and Rouzbahman M: Endometrial stromal tumours revisited: An update based on the 2014 WHO classification. J Clin Pathol 68: 325-332, 2015.

2. Prat J and Mbatani: Uterine sarcomas. Int J Gynaecol Obstet 131 (Suppl 2): S105-S110, 2015.

3. Xue WC and Cheung AN: Endometrial stromal sarcoma of uterus. Best Pract Res Clin Obstet Gynaecol 25: 719-732, 2011.

4. Oliva E, Clement PB and Young RH: Endometrial stromal tumors: An update on a group of tumors with a protean phenotype. Adv Anat Pathol 7: 257-281, 2000.

5. Cohen I: Endometrial pathologies associated with postmenopausal tamoxifen treatment. Gynecol Oncol 94: 256-266, 2004.

6. Ashraf-Ganjoei T, Behtash N, Shariat M and Mosavi A: Low grade endometrial stromal sarcoma of uterine corpus, a clinico-pathological and survey study in 14 cases. World J Surg Oncol 4: 50, 2006.

7. Pekindil G, Tuncyurek O, Orguc S, Inceboz U, Kandiloglu AR and Caglar H: A case of endometrial stromal sarcoma with curvilinear calcification. Gynecol Oncol 98: 318-321, 2005.

8. Aubry MC, Myers JL, Colby TV, Leslie KO and Tazelaar HD: Endometrial stromal sarcoma metastatic to the lung: A detailed analysis of 16 patients. Am J Surg Pathol 26: 440-449, 2002.

9. Itoh T, Mochizuki M, Kumazaki S, Ishihara T and Fukayama M: Cystic pulmonary metastases of endometrial stromal sarcoma of the uterus, mimicking lymphangiomyomatosis: A case report with immunohistochemistry of HMB45. Pathol Int 47: 725-729, 1997.

10. Kim GY, Sung CO, Han J, Park JO and Lee KS: Pulmonary metastases of uterine endometrial stromal sarcoma: Diffuse micronodular and ground glass opacities: A case report. J Korean Med Sci 19: 901-903, 2004.

11. Abrams J, Talcott J and Corson JM: Pulmonary metastases in patients with low-grade endometrial stromal sarcoma. Clinicopathologic findings with immunohistochemical characterization. Am J Surg Pathol 13: 133-140, 1989.

12. Inayama $Y$, Shoji A, Odagiri S, Hirahara F, Ito T, Kawano $\mathrm{N}$ and Nakatani Y: Detection of pulmonary metastasis of low-grade endometrial stromal sarcoma 25 years after hysterectomy. Pathol Res Pract 196: 129-134, 2000.

13. Qian ZD, Huang LL and Zhu XM: An immunohistochemical study of CD83- and CD1a-positive dendritic cells in the decidua of women with recurrent spontaneous abortion. Eur J Med Res 20: 2, 2015.

14. Satoh Y, Ishikawa Y, Miyoshi T, Mukai H, Okumura S and Nakagawa K: Pulmonary metastases from a low-grade endometrial stromal sarcoma confirmed by chromosome aberration and fluorescence in-situ hybridization approaches: A case of recurrence 13 years after hysterectomy. Virchows Arch 442: 173-178, 2003.

15. Murakami A, Hayashi T, Terao Y, Mori T, Kumasaka T, Seyama K and Takahashi K: Cystic, nodular and cavitary metastases to the lungs in a patient with endometrial stromal sarcoma of the uterus. Intern Med 53: 1001-1005, 2014.

16. Miyamoto H, Jones CE, Raymond DP, Wandtke JC, Strang JG, Bourne PA, Bonfiglio TA and $\mathrm{Xu} \mathrm{H}$ : Pulmonary metastases from uterine neoplasms after long tumour-free interval: Four cases and review of the literature. Pathology 41: 234-241, 2009.

17. Lim MC, Lee S and Seo SS: Megestrol acetate therapy for advanced low-grade endometrial stromal sarcoma. Onkologie 33: 260-262, 2010.

18. Mahadeva R, Stewart S and Wallwork J: Metastatic endometrial stromal sarcoma masquerading as pulmonary lymphangioleiomyomatosis. J Clin Pathol 52: 147-148, 1999.

19. Akhavan A, Shishebor F, Moghimi M and Binesh F: Endometrial stromal sarcoma of uterus with metastasis to the lung and brain. BMJ Case Rep 2012: pii: bcr2012006954, 2012.

20. Binesh F, Zahir ST, Akhavan A and Bovanlu TR: Endometrial stromal sarcoma of the uterus presenting as pulmonary metastasis. BMJ Case Rep 2013: pii: bcr2013008565, 2013.

21. Kang DO, Choi SI, Oh JY, Sim JK, Choi JH, Choo JY, Hwang JW, Lee SH, Lee JH, Lee KY, et al: Endometrial stromal sarcoma presented as an incidental lung mass with multiple pulmonary nodules. Tuberc Respir Dis (Seoul) 76: 131-135, 2014.

22. Ota S, Shinagawa K, Ueoka H, Tada S, Tabata M, Hamazaki S, Kondo E, Kiura K, Mannami T, Shibayama T, et al: Spontaneous regression of metastatic endometrial stromal sarcoma. Jpn J Clin Oncol 32: 71-74, 2002. 
23. Steele SJ, Scott JM and Stephens TW: Endometrial stromal sarcoma. Report of a case with a pulmonary metastasis extending through the heart. Br J Surg 55: 943-945, 1968.

24. Nakamura K, Nakayama K, Ishikawa M, Ishikawa N, Katagiri H, Katagiri A, Ishibashi T, Sato E, Iida K, Sultana R and Kyo S: Letrozole as second-line hormonal treatment for recurrent low-grade endometrial stromal sarcoma: A case report and review of the literature. Oncol Lett 12: 3856-3860, 2016.

25. Giin Chong S, Mitchell P, Fabre A and McDonnell TJ: Recurrent pneumothoraces in a 65-year-old female: An unusual case of cystic lung disease. Eur Respir Rev 23: 271-272, 2014.

26. Spano JP, Soria JC, Kambouchner M, Piperno-Neuman S, Morin F, Morere JF, Martin A and Breau JL: Long-term survival of patients given hormonal therapy for metastatic endometrial stromal sarcoma. Med Oncol 20: 87-93, 2003.

27. Hwang H, Matsuo K, Duncan K, Pakzamir E, Pham HQ, Correa A Fedenko A and Mhawech-Fauceglia P: Immunohistochemical panel to differentiate endometrial stromal sarcoma, uterine leiomyosarcoma and leiomyoma: Something old and something new. J Clin Pathol 68: 710-717, 2015.

28. Conklin CM and Longacre TA: Endometrial stromal tumors: The new WHO classification. Adv Anat Pathol 21: 383-393, 2014.

29. Micci F, Panagopoulos I, Bjerkehagen B and Heim S: Consistent rearrangement of chromosomal band $6 \mathrm{p} 21$ with generation of fusion genes JAZF1/PHF1 and EPC1/PHF1 in endometrial stromal sarcoma. Cancer Res 66: 107-112, 2006.

30. Micci F, Gorunova L, Gatius S, Matias-Guiu X, Davidson B, Heim S and Panagopoulos I: MEAF6/PHF1 is a recurrent gene fusion in endometrial stromal sarcoma. Cancer Lett 347: 75-78, 2014.

31. Panagopoulos I, Thorsen J, Gorunova L, Haugom L, Bjerkehagen B, Davidson B, Heim S and Micci F: Fusion of the ZC3H7B and BCOR genes in endometrial stromal sarcomas carrying an X;22-translocation. Genes Chromosomes Cancer 52: 610-618, 2013.
32. Dewaele B, Przybyl J, Quattrone A, Finalet Ferreiro J, Vanspauwen V, Geerdens E, Gianfelici V, Kalender Z, Wozniak A, Moerman P, et al: Identification of a novel, recurrent MBTD1-CXorf67 fusion in low-grade endometrial stromal sarcoma. Int J Cancer 134: 1112-1122, 2014.

33. Amant F, Floquet A, Friedlander M, Kristensen G, Mahner S, Nam EJ, Powell MA, Ray-Coquard I, Siddiqui N, Sykes P, et al: Gynecologic cancer InterGroup (GCIG) consensus review for endometrial stromal sarcoma. Int J Gynecol Cancer 24 (Suppl 3): S67-S72, 2014.

34. Koh WJ, Greer BE, Abu-Rustum NR, Apte SM, Campos SM, Cho KR, Chu C, Cohn D, Crispens MA, Dizon DS, et al: Uterine Sarcoma, Version 1.2016: Featured updates to the NCCN guidelines. J Natl Compr Canc Netw 13: 1321-1331, 2015.

35. Denschlag D, Thiel FC, Ackermann S, Harter P, Juhasz-Boess I, Mallmann P, Strauss HG, Ulrich U, Horn LC, Schmidt D, et al: Sarcoma of the Uterus. Guideline of the DGGG (S2k-Level, AWMF registry No. 015/074, August 2015). Geburtshilfe Frauenheilkd 75: 1028-1042, 2015

36. Yamaguchi M, Erdenebaatar C, Saito F, Motohara T, Miyahara Y, Tashiro $\mathrm{H}$ and Katabuchi H: Long-term outcome of aromatase inhibitor therapy with Letrozole in patients with advanced low-grade endometrial stromal sarcoma. Int J Gynecol Cancer 25: 1645-1651, 2015.

37. Cuppens T, Tuyaerts S and Amant F: Potential therapeutic targets in uterine sarcomas. Sarcoma 2015: 243298, 2015.

This work is licensed under a Creative Commons Attribution-NonCommercial-NoDerivatives 4.0 International (CC BY-NC-ND 4.0) License. 